\title{
Preoperative PET/CT Helps Decide the Extent of Surgery for Medullary Thyroid Cancer When Basal Calcitonin Is $\geq 1000 \mathrm{pg} / \mathrm{ml}$
}

\section{Martin Biermann, ${ }^{1,2}$ Katrin Brauckhoff ${ }^{3}$}

\author{
${ }^{1}$ Nuclear Medicine/PET-Center, Department of Radiology, Haukeland University Hospital, Bergen, Norway \\ ${ }^{2}$ Department of Clinical Medicine, University of Bergen, Bergen, Norway \\ ${ }^{3}$ Endocrine Surgery, Haukeland University Hospital, Bergen, Norwa
}

Review of: Brammen L, Niederle MB, Riss P, Scheuba C, Selberherr A, Karanikas G, Bodner G, Koperek O, Niederle B 2018 Medullary thyroid carcinoma: do ultrasonography and F-DOPA-PET-CT influence the initial surgical strategy? Ann Surg Oncol 25:3919-3927. PMID: 30306375.

\section{SUMMARY}

\section{Background}

Medullary thyroid cancer (MTC) is a neuroendocrine tumor originating from the parafollicular $\mathrm{C}$ cells, which produce the proteohormone calcitonin. MTC accounts for about 2 to $3 \%$ of all thyroid neoplasms. The only curative approach to date is radical surgery (1). At the time of diagnosis, one third of MTC patients show lymph node or distant metastases. Exact preoperative staging may help in selecting a stage-adapted surgical strategy. Neck ultrasound is the primary imaging method used to assess local extent and lymphatic spread (1). As a neuroendocrine tumor, MTC is able to take up and decarboxylate dopamine. Positron-emission tomography (PET) with fluorine-18 labeled dihydroxyphenylalanine (F-DOPA) is the preferred functional imaging method for detecting recurrent or persistent MTC after initial surgery in patients with a basal calcitonin level $>150 \mathrm{pg} / \mathrm{ml}$ (1). Two recent studies have assessed the value of F-DOPA-PET before initial surgery $(2,3)$ one-third of medullary thyroid carcinoma (MTC.

\section{Methods}

The study at the University of Vienna in Austria extends a previously published series of 32 patients with newly diagnosed MTC (3) and included 50 patients who fulfilled the biochemical criteria of MTC after routine calcitonin screening.

Under the screening policy, serum calcitonin was determined in all patients with thyroid nodules regardless of serum thyroid function. A basal calcitonin of $\geq 8 \mathrm{pg} / \mathrm{ml}$ for males or $\geq 6 \mathrm{pg} / \mathrm{ml}$ for females in combination with a calcium-stimulated calcitonin level of $>100 \mathrm{pg} / \mathrm{ml}$ were considered highly suspicious for MTC and were an indication for surgery.

A standardized neck ultrasound of the cervical and supraventricular regions was performed by one radiologist who was aware of the biochemical diagnosis on a General Electric LOGIQ E9 ultrasound machine with a 9-MHz probe.

PET scanning was performed 60 minutes after the intravenous administration of F-DOPA ( $3 \mathrm{MBq} / \mathrm{kg}$ of body weight) on a Siemens Biograph TruePoint 64 hybrid scanner in combination with contrast-enhanced computed tomography (CECT). Lymph nodes were considered suspicious for malignancy when they had visually higher DOPA uptake than 
Martin Biermann and

Katrin Brauckhoff the surrounding background activity or when they had a round shape and showed contrast enhancement (3).

All patients underwent total thyroidectomy with bilateral central neck dissection (level VI) and bilateral lateral lymphadenectomy (level II-V) sparing vessels, muscles, and nerves. In patients with positive F-DOPA PET/CT imaging in the mediastinum but negative imaging for distant metastases, mediastinal lymph node dissection was conducted. In patients with distant metastases, a less radical lateral lymph node dissection was performed.

Histopathologic studies, including immunohistochemistry, were performed for all macroscopically suspect lesions by a single pathologist who was aware of the elevated basal calcitonin levels.

\section{Results}

The mean age of the 50 patients was 57 years (range, 9-79), and 26 of the patients were female. Basal serum calcitonin was $202 \mathrm{pg} / \mathrm{ml}$ (range, 82-1074). Seventeen patients were UICC stage pT1a (tumor diameter $\leq 10 \mathrm{~mm}), 15 \mathrm{pT} 1 \mathrm{~b}(11-20 \mathrm{~mm})$, nine pT2 (21-40 mm), and nine pT3 (>40 mm, or with minimal extrathyroidal extension). Eight MTCs were multifocal, and eight were hereditary. Twenty-one patients had lymph node metastases (18 central, 16 lateral, and 3 mediastinal). A mean ( \pm SD) of $74 \pm 7$ lymph nodes were removed per patient, and $6.7 \pm 2$ of these were positive. Three patients had distant metastases. Seven patients suffered temporary hypoparathyroidism. Temporary recurrent laryngeal-nerve paralysis occurred in 4 of 100 nerves at risk. There were no permanent complications. Biochemical cure was achieved in 34 of 50 patients (68\%). Five patients died of progressive MTC after a mean follow-up of $60 \pm 31$ months.
Neck ultrasound was true positive for detection of the primary tumor in 45 of 50 patients and F-DOPA $\mathrm{PET} / \mathrm{CT}$ in 43 of 50 , with a sensitivity of $90 \%$ and $86 \%$, respectively.

Lymph node metastases were detected on ultrasound in 9 of 21 patients and F-DOPA-PET/CT in 12 of 21 patients, yielding a sensitivity of $43 \%$ and $57 \%$, respectively. For diagnosing central lymph node metastases, ultrasound was true positive in only 1 of 18 patients and F-DOPA PET/CT in 5 of 18 patients, with a sensitivity of $6 \%$ and $28 \%$, respectively. In the lateral neck, neck ultrasound was true positive in 9 of 16 patients and false positive in 1 (sensitivity, 56\%; specificity, 97\%), while F-DOPA-PET/CT was true positive in 12 of 16 patients with no false positives (sensitivity, $75 \%$; specificity, $100 \%$ ). In a subanalysis of 11 patients with 1 to 10 lymph node metastases, the sensitivity of ultrasound was $9 \%$ and of F-DOPA PET/CT 27\%.

F-DOPA-PET/CT was true positive in three patients with mediastinal lymph node metastases. None of the patients with mediastinal lymph node metastases had distant metastases, and basal calcitonin values were above $12,000 \mathrm{pg} / \mathrm{ml}$. Three patients had distant metastases to the liver only $(n=2)$ or to the lungs, bone, and liver ( $n=1)$, all with basal calcitonin values above $1900 \mathrm{pg} / \mathrm{ml}$.

\section{Conclusions}

In newly diagnosed MTC, neither ultrasound nor F-DOPA PET/CT is sensitive for the presence and location of lymph node metastases in the central and lateral neck. Preoperative F-DOPA-PET/CT is most useful for the diagnosis of mediastinal lymph node metastasis and distant metastases in patients with a basal calcitonin $\geq 500-1000 \mathrm{pg} / \mathrm{ml}$. 
Martin Biermann and

Katrin Brauckhoff

\section{COMMENTARY}

The only curative approach to treating MTC is surgery (1). The current guidelines of the American Thyroid Association recommend total thyroidectomy and dissection of the lymph nodes of the central compartment (level VI). Dissection of the ipsilateral and contralateral lateral compartments (levels II-V) is recommended dependent on preoperative imaging and/or preoperative serum calcitonin (1).

As shown in a series of 715 patients operated on at a single institution, the number of metastatic lymph nodes is a major prognostic indicator of MTC (4). In patients with $\leq 10$ metastatic lymph nodes, the risk of distant metastases (especially in the lung) is less than $4 \%$, and surgical cure is possible (4). It is therefore appealing to attempt to improve preoperative imaging for the identification of cervical and upper mediastinal lymph node metastases so that the extent of surgery can be better adapted to the risk profile of the patient. However, in the subgroup of patients with $\leq 10$ lymph node metastases in this study, sensitivity of both ultrasound and F-DOPAPET/CT were disappointing. The authors concluded that in patients in whom only a few or microscopic lymph node metastases are expected, F-DOPAPET/CT was of no practical diagnostic value.

The chief value of F-DOPA-PET/CT was for the diagnosis of mediastinal lymph node metastases and for distant metastases. In the former situation, mediastinal lymph node dissection was performed, while in the latter condition, radicality of the lateral neck dissection was reduced. This affected 6 of 50 patients in the series, all of whom had a basal calcitonin level in excess of $1000 \mathrm{pg} / \mathrm{ml}$.

The limited sensitivity of ultrasound for the detection of central lymph node metastases has been described in many studies of thyroid cancer of follicular cell origin and agrees with our own experience (5). However, contrast-enhanced CT is highly useful, particularly when it is combined with ultrasound performed by the same operator. Regrettably, the authors did not include a separate analysis of the performance of CECT alone. It is unclear how many of the six patients with mediastinal or distant metastases could have been correctly diagnosed based on CECT alone. Of note, in the initial publication from the same institution, all patients with a basal calcitonin level $>500 \mathrm{pg} / \mathrm{ml}$ (14 of 32 patients) underwent magnetic resonance imaging (MRI) of the liver (3), which presumably would have identified all three patients harboring distant metastases in this series.

F-DOPA is the tracer of choice for detecting recurrent MTC after initial radical surgery. When available, it is recommended in patients with the basal calcitonin above $150 \mathrm{pg} / \mathrm{ml}$ (1). Based on the data from the current series, it is too early to conclude the extent to which F-DOPA PET/CT should be included in the preoperative workup of patients with suspected MTC. However, improving preoperative workup, rather than spending the same resources for diagnosing recurrences, is laudable. Unfortunately, the availability of F-DOPA is still very limited in most countries, including our own. We have adopted a similarly aggressive imaging strategy for the preoperative workup of thyroid cancer of follicular-cell origin, including preoperative PET/CT using 18F- fluorodeoxyglucose (18F-FDG). This approach modifies our intended surgical approach in about $20 \%$ of our patients not only with recurrent thyroid cancer (5), but also in our newly diagnosed patients. 
Martin Biermann and

Katrin Brauckhoff
The present study has the following limitations: (i) The cohort included patients detected through serum calcitonin screening and thus a high proportion of patients with early-stage disease. (ii) All patients except those with distant metastases underwent modified radical bilateral neck dissection. This approach is more radical than that recommended by the current ATA guidelines. While this provides histopathology as a gold standard for evaluating the performance of the diagnostic methods, it makes it difficult to show the potential therapeutic benefit of more extended diagnostic imaging. (iii) Only patients with positive medi- astinal F-DOPA-PET/CT underwent mediastinal lymph node detection. The sensitivity of F-DOPAPET of $100 \%$ is most probably overestimated since the rate of false negative examinations cannot be ascertained.

In conclusion, F-DOPA-PET/CT allows the timely diagnosis of mediastinal lymph node metastases and distant metastases in the preoperative workup of patients with MTC, while the sensitivity for detecting lymph node metastases in the central and lateral neck is limited.

\section{References}

1. Wells SA, Asa SL, Dralle H, Elisei R, Evans DB, Gagel RF, Lee N, Machens A, Moley JF, Pacini F, et al. 2015 Revised American Thyroid Association guidelines for the management of medullary thyroid carcinoma. Thyroid 25:567-610.

2. Brammen L, Niederle MB, Riss P, Scheuba C, Selberherr A, Karanikas G, Bodner G, Koperek O, Niederle B 2018 Medullary thyroid carcinoma: do ultrasonography and F-DOPA-PET-CT Influence the initial surgical strategy? Ann Surg Oncol 25:39193927.

3. Rasul S, Hartenbach S, Rebhan K, Göllner A, Karanikas G, Mayerhoefer M, Mazal P, Hacker M, Hartenbach M 2018 [18F]DOPA PET/ceCT in diagnosis and staging of primary medullary thyroid carcinoma prior to surgery. Eur J Nucl Med Mol Imaging 45:2159-2169.
4. Machens A, Dralle H 2013 Prognostic impact of $\mathrm{N}$ staging in 715 medullary thyroid cancer patients: proposal for a revised staging system. Ann Surg 257:323-329.

5. Biermann M, Kråkenes J, Brauckhoff K, Haugland HK, Heinecke A, Akslen LA, Varhaug JE, Brauckhoff M 2015 Post-PET ultrasound improves specificity of 18F-FDG-PET for recurrent differentiated thyroid cancer while maintaining sensitivity. Acta Radiol 56:1350-1360. 